\title{
Bose-Einstein correlations for Lévy stable source distributions
}

\author{
T. Csörgo", ${ }^{a, 1}$ S. Hegyi a,2 and W. A. Zajc ${ }^{\text {b,3 }}$ \\ ${ }^{a}$ KFKI RMKI, H-1525 Budapest 114, POB 49, Hungary \\ ${ }^{\mathrm{b}}$ Dept. Physics, Columbia University, $538 \mathrm{~W} 120$ th St, NY 10027 New York
}

\begin{abstract}
The peak of the two-particle Bose-Einstein correlation functions has a very interesting structure. It is often believed to have a multivariate Gaussian form. We show here that for the class of stable distributions, characterized by the index of stability $0<\alpha \leq 2$, the peak has a stretched exponential shape. The Gaussian form corresponds then to the special case of $\alpha=2$. We give examples for the Bose-Einstein correlation functions for univariate as well as multivariate stable distributions, and check the model against two-particle correlation data.
\end{abstract}

Key words: correlations, elementary particle physics, heavy ion physics, statistical analysis, Lévy-stable distributions

\section{Introduction}

The structure of the peak of two-particle Bose-Einstein correlation functions (BEC-s) is a very interesting physically measurable quantity. It carries information about the fraction of long-lived resonances [1], hence signals possible $U_{A}(1)$ symmetry restoration in high energy heavy ion reactions [2], it can be utilized to determine a generalized Hubble constant for the transverse flow in high energy heavy ion physics [3] and it is also sensitive to a possible partial coherence in the source [4], for other examples see the recent reviews of refs. $[5,6]$.

\footnotetext{
1 Email: csorgo@sunserv.kfki.hu

$2 \quad$ hegyi@rmki.kfki.hu

3rzajc@nevis.columbia.edu
} 
Originally, the method of Bose-Einstein correlations was invented by the radio astronomers R. Hanbury Brown and R. Q. Twiss (HBT), who applied it to determine the angular diameters of main sequence stars $[7,8]$. They referred to the method as intensity interferometry. In particle physics, an analogous effect was discovered independently [9] by G. Goldhaber, S. Goldhaber, W. Y. Lee and A. Pais (GGLP), who observed an enhancement of identical pions with small opening angles in $p+\bar{p}$ reactions. Essentially, the enhancement of identical bosons with small momentum difference is due to their Bose-Einstein (BE) statistics. The effect is frequently referred to as either the HBT, or the GGLP effect, or intensity interferometry, or simply as BE correlations (BEC).

The detailed shape analysis of the BEC is rather complicated but important, because the shape carries information about the space-time structure of the particle emission process. Recently, two of us proposed [10] a modelindependent method for the analysis of the shape of this function, based on an experimentally preferred weight function and a complete orthogonal set of polynomials, where orthogonality is defined with respect to a preferred weight function. In the case of approximately Gaussian correlation functions, the method results in the Edgeworth expansions, and the complete orthogonal set of functions are the Hermite polynomials. In the case of an approximately exponential shape, the expansion is given in terms of the Laguerre polynomials. For approximately spherical distributions the expansion can be formulated in terms of spherical harmonics (see ref. [10] for further details).

The Edgeworth and the Laguerre expansions are very general methods to characterize the two-particle correlation functions. They depend only on the following two

experimental conditions:

i) The correlation function tends to a constant for large values of the relative momentum $Q$.

ii) The correlation function deviates from its asymptotic, large $Q$ value in a certain domain of its argument.

For simplicity, this domain, where the correlation function deviates from its asymptotic value, with other words, the location of its non-trivial structure, is assumed to be close to $Q=0$.

This implies that the Edgeworth, Laguerre (and similar) expansions can be applied to all kinds of data that satisfy properties i) and ii), as the bosonic properties of the observed particles are not utilized. Such expansions are useful if one would like to study and characterize short range correlations in a general manner, and if no attempt is made to connect the structure of the shape of the BEC to the characteristics of the underlying space-time picture. (The 
conditions for the convergence of such expansions are given in ref. [10].)

The purpose of the present study is to provide a model-independent characterization of the BEC with a further additional assumption:

iii) The two-particle correlation function is related to a Fourier transformed space-time distribution of the source.

This last assumption implies that the plane-wave approximation can be warranted, and additional possible short-range correlations due to e.g. the final state Coulomb or strong interactions as well as energy-momentum conservation or the jet structure of the source can be removed from the data or their magnitude is under theoretical and/or experimental control.

The purpose of the present paper is to determine in a general form the approximate behaviour of the two-particle BEC under assumptions i) - iii) and relate the parameters of the source functions to the parameters of the BEC in a generic form.

\subsection{Basics}

The two-particle BEC is defined as the ratio of the two-particle invariant momentum distribution $N_{2}\left(\mathbf{k}_{1}, \mathbf{k}_{2}\right)$ to the product of the single-particle invariant momentum distributions $N_{1}\left(\mathbf{k}_{i}\right)$ [11], where $\mathbf{k}$ stands for the three-momentum

of the detected particle of mass $m$ and energy $E=\sqrt{m^{2}+\mathbf{k}^{2}}$. The BECF is defined as

$$
C_{2}\left(\mathbf{k}_{1}, \mathbf{k}_{2}\right)=\frac{N_{2}\left(\mathbf{k}_{1}, \mathbf{k}_{2}\right)}{N_{1}\left(\mathbf{k}_{1}\right) N_{1}\left(\mathbf{k}_{2}\right)},
$$

for more details on the normalizations see refs. [5,11]. For simplicity, we assume that at large relative momenta, the two-particle correlation function tends to 1. Thus we neglect possible long-range correlations and normalization coefficients in eq. (1).

As $C_{2}\left(\mathbf{k}_{1}, \mathbf{k}_{2}\right)$ is a ratio of invariant momentum distributions, the correlation function is also an invariant observable. This property can be emphasized by using the $C_{2}\left(k_{1}, k_{2}\right)$ notation, where $k_{1}$ and $k_{2}$ are the momentum four-vectors of the particle pair. See also refs. [5,6] for more detailed discussions about the constraints between $k_{1}$ and $k_{2}$.

We assume that $C_{2}\left(k_{1}, k_{2}\right)$ is experimentally determined and satisfies properties i) - iii). These correlation functions can be evaluated at a certain fixed mean momentum $\mathbf{K}=0.5\left(\mathbf{k}_{1}+\mathbf{k}_{2}\right)$ as a function of various components of the 
relative momentum $\mathbf{q}=\mathbf{k}_{1}-\mathbf{k}_{2}$. The relative momentum can be decomposed into some experimentally preferred relative momentum components in 1, 2 or 3 dimensions. For example, experimental data are presented in one dimension in terms of the invariant momentum difference $Q_{I}=\sqrt{-\left(k_{1}-k_{2}\right)^{2}}$, or as a function of various components of the relative momentum, for example, $\left(q_{0}, q_{z}, q_{t}\right)=\left(E_{1}-E_{2}, \mathbf{k}_{1, z}-\mathbf{k}_{2, z}, \sqrt{\left(\mathbf{k}_{1, x}-\mathbf{k}_{2, x}\right)^{2}+\left(\mathbf{k}_{1, y}-\mathbf{k}_{2, y}\right)^{2}}\right)$.

Various directional decompositions of the relative momentum with respect to the mean momentum have been proposed. These decompositions include the Bertsch-Pratt, the Yano-Koonin-Podgoretskii and the invariant Buda-Lund decompositions. For further details, we refer the interested readers to the recent reviews on these topics, [5,6,12-14]. For our further considerations, the specific experimental choice for the directional decomposition of the relative momenta will not be essential.

Essential background information on the generic structure of the peak of the BEC was summarized in ref. [15] regarding the impossibility of the Taylor expansion of the correlation functions around the $Q=0$ point, and in the appendix of ref. [16], where it was pointed out that the class of the stable distributions plays an important role in non-Gaussian BEC studies. The breakdown of the Gaussian approximation around the $Q=0$ point was shown with the help of a theoretical analysis and a numerical simulation in ref. [17]. Explicit simulations, including long tailed and/or asymmetric source density distributions confirmed this result [18].

The above results are essentially the consequence of the fact that the twoparticle phase-space volume grows as $Q^{3}$ for small values of the relative momentum $Q$. Hence the number of pion pairs tends to zero as $Q \rightarrow 0$, which implies that the numerator and the denominator of eq. (1) tend to 0 in the same limit, $\lim _{Q \rightarrow 0} C_{2}(Q)=$ "0/0". This is the reason why it is experimentally impossible to determine $C(Q=0)$ exactly: the experimental errors on the correlation function diverge as $Q \rightarrow 0$. (Not to mention $\frac{d C(Q)}{d Q}$ and $\frac{d^{2} C(Q)}{d Q^{2}}$ whose experimental errors diverge even more than that of $C(Q))$. So the two-particle correlation function can only be measured at non-zero values of $Q>Q_{\text {min }}$, at a scale set by the combination of factors such as the available statistics, the two-track resolution and the extent to which final-state interactions are under control. Typical values of $Q_{\text {min }}$ are $5-10 \mathrm{MeV}$ in present correlation measurements in heavy ion physics. The value of the Bose-Einstein correlation function at $Q=0$ can then be estimated with various extrapolation techniques, e.g. with the help of a (typically Gaussian) model assumption for the functional form of $C(Q)$, or with the help of model-independent Edgeworth or Laguerre expansions [10]. 


\section{Bose-Einstein correlations for univariate stable distributions}

For clarity, let us consider first the case of a one-dimensional static boson source, and show the essential ideas in the simplest possible manner. (In the subsequent sections we generalize these results to more realistic, expanding sources that contain correlations between coordinate space and momentum space, and also to the case of more complicated derivations.)

Thus we assume here that the source can be characterized by a factorized form of the emission function:

$$
S(x, k)=f(x) g(k)
$$

where $x$ and $k$ are one-dimensional coordinate and momentum variables, respectively. The normalization is chosen such that

$$
\int \mathrm{d} x f(x)=1, \quad \int \mathrm{d} k g(k)=\langle n\rangle,
$$

where $\langle n\rangle$ stands for the mean multiplicity. The single-particle spectrum is obtained as

$$
N_{1}(k)=\int \mathrm{d} x S(x, k)=g(k) .
$$

Assuming chaotic particle emission, and the validity of the plane-wave approximation, the two-particle Bose-Einstein symmetrized wave-function reads as

$$
\psi_{k_{1}, k_{2}}\left(x_{1}, x_{2}\right)=\frac{1}{\sqrt{2}}\left[\exp \left(i k_{1} x_{1}+i k_{2} x_{2}\right)+\exp \left(i k_{1} x_{2}+i k_{2} x_{1}\right)\right] .
$$

In the Yano-Koonin formalism [20], the two-particle momentum distribution is determined as

$$
N_{2}\left(k_{1}, k_{2}\right)=\int \mathrm{d} x_{1} \mathrm{~d} x_{2} S\left(x_{1}, k_{1}\right) S\left(x_{2}, k_{2}\right)\left|\psi_{k_{1}, k_{2}}\left(x_{1}, x_{2}\right)\right|^{2},
$$

and the resulting two-particle BEC is found to be

$$
C_{2}\left(k_{1}, k_{2}\right)=1+\left|\tilde{f}\left(q_{12}\right)\right|^{2},
$$

which measures the absolute value squared Fourier transformed coordinatespace distibution function of the particle emitting source, where the Fourier 
transformed source density (often referred to as the characteristic function) and the relative momentum are defined as

$$
\tilde{f}\left(q_{12}\right)=\int \mathrm{d} x \exp \left(i q_{12} x\right) f(x), \quad q_{12}=k_{1}-k_{2} .
$$

As the two-particle Bose-Einstein correlation function is determined by the modulus of this Fourier-transformed source distribution, but is insensitive to the phase of the Fourier transform, these modulus squared Fourier transforms measure only the relative coordinate distributions of the source, but they are not sensitive to shift parameters like the location of the center of the source distribution in space-time. It is frequently assumed (either explicitly or implicitly), that the function $\tilde{f}(q)$ is analytic around $q=0$ and that its second order Taylor expansion characterizes its behaviour well even for large values of $q$. We shall see later that this imposes severe constraints on the possible choice of the source functions. As this point is essential, we emphasize that the analyticity of $\tilde{f}(q)$ at $q=0$ is an assumption that greatly reduces the possible forms of the source densities. This may be clearly seen in the case of Levy stable distributions[21-23], which are characterized by four real parameters, and thus form an uncountably infinite set. The most essential of these four parameters is the index of stability $\alpha$, which may take any value in the interval $(0,2]$. However, the assumption that the $f(q)$ characteristic function is analytic restricts this parameter to the special $\alpha=2$ case, corresponding to Gaussian Bose-Einstein correlation functions.

If the function $\tilde{f}(q)$ is an analytic function at $q=0$ and if the lhs of eq. (8) can be expanded into a convergent series, one obtains

$$
\tilde{f}(q) \approx 1+i q\langle x\rangle-q^{2}\left\langle x^{2}\right\rangle / 2+\ldots
$$

where

$$
\left\langle x^{n}\right\rangle=\int \mathrm{d} x x^{n} f(x) .
$$

This, in turn, implies that

$$
C(q)=1+|\tilde{f}(q)|^{2} \approx 2-q^{2}\left(\left\langle x^{2}\right\rangle-\langle x\rangle^{2}\right) \approx 1+\exp \left(-q^{2} R^{2}\right),
$$

where a Gaussian source radius parameter $R$ is defined as the width of the source emission function,

$$
R=\sqrt{\left\langle x^{2}\right\rangle-\langle x\rangle^{2}}
$$


Such type of over-simplified derivations, repeated in multi-dimensional forms, are frequently utilized to "prove" that the Bose-Einstein correlation function "has to have a Gaussian form", without investigating the domain of applicability of the approximations that yield this result.

In fact, a Gaussian ansatz for the two-particle BEC frequently gives a good first approximation to the data, in particular, if the statitistical precision of the date is not adequate to perform a detailed shape analysis. One of the reasons for such a behaviour could be that the emission of elementary particles is a rather complicated, stochastic process, in particular in collisions with very high energies. If one assumes that there are many independent processes, that shift by $\delta x$ the coordinate $x$, and that the the final production point is a sum of many, similarly distributed, random shifts, $x=\sum_{i} \delta x_{i}$, and the shifts are characterized by finite variances, then for such random variables the probability distribution for $x$ tends to a Gaussian one, if all the conditions of the Central Limit Theorem are satisfied. As the Fourier transform of a Gaussian is also Gaussian in such cases the expected shape of the two-particle BEC is also a Gaussian.

However, there are many random processes for additive random variables, where a limiting distribution exists, but the Gaussian version of the central limit theorem is not applicable. Such processes are characterized by large fluctuations, power-law like tails and the non-analytic behaviour of the characteristic function of the probability distribution for small values of its arguments. In mathematical statistics and probability theory, such distributions are frequently referred to as Lévy, or stable distributions.

Let us approach the problem of the shape of the two-particle BEC without a reference to a particular model, from the more general point of view of mathematical statistics and the (generalized) Central Limit Theorems. Concrete, model specific applications shall be presented elsewhere [24].

\section{Lévy stable distributions and generalized central limit theorems}

In physics, as well as in the theory of probability, the probability distribution of a sum of a large number of random variables is one of the important problems, as such distributions are frequently realized in Nature. Limit distributions characterize the probability distributions of random processes in the limiting

case when number of the elementary independent random subprocesses tends to infinity.

Various forms of the Central Limit Theorem state, that under some conditions, the sum of a large number of random variables behaves as a Gaussian 
distribution. Essentially, the conditions for the validity of the various central limit theorems are that the elementary distributions should have a finite mean and variance. The sum of a large number of such variables follows a Gaussian distribution, which in turn is a (frequently encountered) special case of limit distributions.

For example, rapidity is a kinematic variable invariant under longitudinal Lorentz transformations. The final (observed) rapidity of a particle is a consequence of many random and independent actions, which can be considered as some rapidity shifts. In case of rescattering on a hadronic or on a partonic medium, the final rapidity of the particles is obviously a sum of many random rapidity shifts. If these subprocesses have finite means and variances, then the central limit theorem implies that the observable rapidity distribution has to be a Gaussian, regardless of the details of the probability distribution of rapidity shifts in the elementary collisions.

The stable distributions are precisely those limit distributions that can occur in Generalized Central Limit theorems. Their study was begun by the mathematician Paul Lévy in the 1920's. A recent book by Zolotarev and Uchaikin [21] contains over 200 pages of applications of stable distributions in probabilistic models, correlated systems and fractals, anomalous diffusion and chaos, physics, radiophysics, astrophysics, stochastic algorithms, financial applications, biology and geology. Stable distributions provide solutions to certain ordinary and fractional differential equations, and the breadht of their applications suggests that they can be considered as a class of special functions [21-23].

\subsection{Characteristic functions}

The Fourier transformed density distribution, eq. (8) is usually called the characteristic function in mathematical statistics. The stable distributions are frequently given in terms of their characteristic functions, as the explicit formulas of the corresponding source density distributions are known only in some special cases.

The characteristic functions of univariate stable distributions can be defined using various convention schemes. In this work, we utilize the notation of Nolan [23]. In this work, the characteristic function of univariate stable distributions is given in three different conventions, out of which we utilize the convention $S(\alpha, \beta, \gamma, \delta ; 1)$ as this leads to natural generalization of data parameterizations utilized before in Bose-Einstein correlations in high energy physics. In this convention, the characteristic function $\tilde{f}(q)$, with other words, the Fourier-transform of a probability density given by the $S(\alpha, \beta, \gamma, \delta ; 1)$ law, 
has the following general form:

$$
\tilde{f}(q)=\exp \left(-\gamma^{\alpha}|q|^{\alpha}+i \beta \gamma^{\alpha}|q|^{\alpha} \operatorname{sign}(q) \tan \left(\frac{\alpha \pi}{2}\right)+i q \delta\right), \quad \alpha \neq 1
$$

Thus univariate stable distributions are characterized by four parameters: the Lévy index of stability (or characteristic exponent) $0<\alpha \leq 2$, the skewness parameter $-1 \leq \beta \leq 1$, the scale parameter $0<\gamma$ and the location parameter $-\infty<\delta<\infty$. We recommend to consult the book of J. Nolan for greater details and the $\alpha=1$ special case, [23], see in particular Fig. 1.3 of this work for an illustration of the stable densities in the $S(\alpha, \beta, \gamma, \delta ; 1)$ parameterization [23].

Before discussing the univariate case in general, let us start with one of the simplest cases. Mathematically, this corresponds to the selection of the symmetric stable distributions. Their relationship with the Gaussian case is also the most apparent. Let us also introduce physical units. Using the notation of Nolan [23], this choice corresponds to the $S\left(\alpha, \beta=0, \gamma=R / 2^{\frac{1}{\alpha}}, \delta=x_{0} ; 1\right)$ convention.

For symmetric stable distributions, the Fourier transformed density distribution has a simple form,

$$
\tilde{f}(q)=\exp \left(i q \delta-|\gamma q|^{\alpha}\right)
$$

where $|z|$ stands for the modulus (absolute value) of the variable $z$, and the support of the density function $f(x)$ is $(-\infty, \infty)$. More complicated cases are discussed in the subsequent parts. In order to simplify the results, and to present results that are similar to the ones used in data fitting in high energy physics, it is useful to re-scale the scale parameter $\gamma$ of the Lévy distributions and introduce a physical notation as follows:

$$
R=2^{\frac{1}{\alpha}} \gamma \quad x_{0}=\delta \quad(\text { if } \quad \beta=0),
$$

In the chosen $S(\alpha, \beta, \gamma, \delta ; 1)$ notational system, for symmetric $(\beta=0)$ stable distributions, the parameter $\delta$ coincides with $x_{0}$, the location parameter of the distribution.

A generic property of the stable distributions is that their characteristic function is non-analytic at $q=0$ for all values of the index of stability $\alpha<2$. For symmetric distributions, the following small $q$ expansion reflects this nonanalytic behaviour:

$$
\tilde{f}(q) \approx 1+i q x_{0}-\frac{1}{2}|q R|^{\alpha}
$$


The analyticity of this expansion is restored only if $\alpha=2$, corresponding to the Gaussian limit.

Lévy-stable distributions yield the following, relatively simple form of the twoparticle BEC:

$$
C(q ; \alpha)=1+\exp \left(-|q R|^{\alpha}\right)
$$

This form that has an additonal parameter, the index of stability $\alpha$, as compared to the usual Gaussian (or exponential) distribution, where the value of $\alpha$ is fixed to 2 (or 1 ).

Note that eq. (14) is not an approximation for the shape of the characteristic function around the $q=0$ point, but an exact and deep mathematical result, a consequence of the Generalized Central Limit Theorem, which gives the possible forms of the Fourier transform of the density distributions of the stable laws. Hence eq. (14) is valid for all values of $q$, and the corresponding form, eq. (17) for the two-particle BEC is well suited for a detailed shape analysis in the physically resolvable large $q$ region.

The simplicity of eq. (17) is the reason why we utilized the $S\left(\alpha, \beta, \gamma, \delta=x_{0} ; 1\right)$ convention with a rescaling of the $\gamma$ parameter to $R$ : In cases of $\alpha=1$ and $\alpha=2$ we obtain formulas that already had a number of applications in particle interferometry studies in high energy physics $[5,6,14]$.

The physical meaning of the index of stability is that the symmetric stable distributions decrease for large values of the coordinate $x$ as a power-law,

$$
f(x) \propto x^{-1-\alpha}, \quad \text { for } \quad x \gg R, \quad 0<\alpha<2,
$$

while in the $\alpha=2$ special case corresponds to Gaussian source distributions.

In general, stable distributions with $0<\alpha<2$ are long tailed and are frequently related to the self-similarity of the generating mechanism. For example, in QCD, the theory of strong interactions, jets emerge within jets within jets etc, a process characterized by a self-similar branching and a fractal structure, where the fractal dimension of the source is related to the anomalous dimension of QCD [26-28].

In the Lund model of hadronization, a self-similar string fragmentation process determines the distribution of the directly produced particles [29]. Among these, there is a big range of the distribution of the decay times of various resonances, and their decay may lead also to a power-law tail fluctuation of the effective source sizes [30]. Numerically, similar results were obtained by 
Utyuzh, Wilk and Włodarczyk in ref. [31] when considering Bose-Einstein correlations for sources with a fractal, power-law structure in space-time.

A second order phase transition is expected if a heavy ion collision passes through the critical point of the quark-gluon plasma - hadronic matter phase transition, throught the end-point of the phase-boundary along which the transition is of first order. In the vincinity of the critical point the second order phase transition is expected to be signalled by power-law type of fluctuations in coordinate space. Coordinate distributions with power-law tails may obey the Generalized Central Limit Theorem, which essentially means that these density distributions, in the limit of large number of independent sources, tend to a Lévy stable distribution with index of stability $\alpha<2$.

\subsubsection{Some examples}

1) The Gaussian distribution corresponds to the case of $\alpha=2$. The scale parameter is $R=\sqrt{\left\langle x^{2}\right\rangle-\langle x\rangle^{2}}$, the standard deviation. The density distribution and the corresponding BEC are

$$
\begin{aligned}
& f(x)=\frac{1}{\left(2 \pi R^{2}\right)^{1 / 2}} \exp \left[-\frac{\left(x-x_{0}\right)^{2}}{2 R^{2}}\right], \quad-\infty<x<\infty, \\
& C(q)=1+\exp \left(-q^{2} R^{2}\right) .
\end{aligned}
$$

2) The Lorentzian (or Cauchy) distribution corresponds to the case of $\alpha=$

1. The scale parameter $R$ is the standard scale parameter of the Lorentz distribution. The density distribution and the corresponding BEC are

$$
\begin{aligned}
f(x) & =\frac{1}{\pi} \frac{R}{R^{2}+\left(x-x_{0}\right)^{2}}, \quad-\infty<x<\infty, \\
C(q) & =1+\exp (-|q R|) .
\end{aligned}
$$

Such a form was frequently applied to describe the $Q_{I}$ dependence of twoparticle BEC in high energy heavy ion and particle physics [14,5].

3) The asymmetric Lévy distribution is a slightly more complicated, but still relatively simple case, corresponding to $\alpha=1 / 2, \beta=1$. The density has the following one-sided distribution

$$
\begin{aligned}
& f(x)=\sqrt{\frac{R}{8 \pi}} \frac{1}{\left(x-x_{0}\right)^{3 / 2}} \exp \left(-\frac{R}{8\left(x-x_{0}\right)}\right), \quad x_{0}<x<\infty, \\
& C(q)=1+\exp (-\sqrt{|q R|}) .
\end{aligned}
$$

This density distribution has been considered, although in a different formalism, for the characterization of two-particle BEC in ref. [15]. 


\subsection{Asymmetric Lévy distributions}

Adopting Nolan's $S(\alpha, \beta, \gamma, \delta ; 1)$ convention for the univariate Lévy stable densities with the rescaling of the scale parameter $\gamma$ to $R$, eq. (15), the characteristic function $\tilde{f}(q)$ has the following general form:

$$
\tilde{f}(q)=\exp \left(-\frac{1}{2} R^{\alpha}|q|^{\alpha}+i \frac{\beta}{2} R^{\alpha}|q|^{\alpha} \operatorname{sign}(q) \tan \left(\frac{\alpha \pi}{2}\right)+i q x_{0}\right), \quad \alpha \neq 1,(25)
$$

and we recommend to consult the book of J. Nolan for the $\alpha=1$ special case, [23].

This form of the Generalized Central Limit Theorem also implies that twoparticle BEC-s, measuring the modulus square of the Fourier transformed source distribution, are insensitive to the asymmetry parameter $\beta$ of the Lévy stable distributions, as well as to the center $x_{0}$ of the source. This is the direct consequence of the fact, that the two-particle Bose-Einstein correlations measure only the relative coordinate distributions, hence asymmetry or the position of the center of the distribution does not enter the observable correlations.

If the source is characterized by a Lévy stable distribution, the two-particle $\mathrm{BEC}$ can always be written in the form of eq. (17) in the whole range of $q$.

Note that for $\beta \neq 0$, the interpretation of the $\gamma$ and $\delta$ parameters of the $S(\alpha, \beta, \gamma, \delta ; 1)$ is not straightforward, and this parameterization is not a continuous function of the parameter $\alpha$ at the $\alpha=1$ point. See ref. [23] for a more detailed discussion on these points.

\subsection{Three-particle Bose-Einstein correlations for stable distributions}

Can one extract, at least in principle, the asymmetry parameter $\beta$ of the stable laws, using the methods of particle interferometry? In order to answer this question, let us consider the three-particle Bose-Einstein correlation function. In general, it may have a complicated form [4,5]. However, if the particle emission is completely chaotic and if the plane-wave approximation can be warranted, then the three-particle BECF reads as follows:

$$
\begin{aligned}
C_{3}(1,2,3)=1+|\tilde{f}(1,2)|^{2}+|\tilde{f}(2,3)|^{2} & +|\tilde{f}(3,1)|^{2}+ \\
& +2 \mathcal{R} \tilde{f}(1,2) \tilde{f}(2,3) \tilde{f}(3,1) .
\end{aligned}
$$


where the symbolic notation $\tilde{f}(i, j) \equiv \tilde{f}\left(k_{i}-k_{j}\right) \equiv \tilde{f}\left(q_{i j}\right)$ has been introduced to simplify the equation.

The three-particle cumulant correlation function corresponds to the last term,

$$
\kappa_{3}(1,2,3)=2 \mathcal{R} \tilde{f}(1,2) \tilde{f}(2,3) \tilde{f}(3,1)
$$

where the two-particle cumulant correlation function is defined as

$$
\kappa_{2}(1,2)=|\tilde{f}(1,2)|^{2}
$$

Hence, the normalized and symmetrized ratio

$$
w(1,2,3)=\frac{\kappa_{3}(1,2,3)}{2 \sqrt{\kappa_{2}(1,2) \kappa_{2}(2,3) \kappa_{2}(3,1)}}
$$

turns out to be a simple function of the asymmetry parameter of the Lévy stable distributions:

$$
w(1,2,3)=\cos \left\{\frac{\beta}{2} R^{\alpha} \tan \left(\frac{\alpha \pi}{2}\right)\left[\sum_{(i, j)}\left|q_{i j}\right|^{\alpha} \operatorname{sign}\left(q_{i j}\right)\right]\right\} \quad \text { for } \quad \alpha \neq 1,
$$

(for the special case of $\alpha=1$, see again ref. [23].) In the above equation, the summation is taken over the cyclic permutations, $(i, j)=(1,2),(2,3)$, or $(3,1)$. Note that the displacement parameter $\delta=x_{0}$ cancels from this result, too. However, this parameter, related to the center of the particle emitting source, is not considered as an essential quantity in the physics of particle interferometry. For simplicity we may assume that the origin of our coordinate system is fixed by the condition $x_{0}=0$.

Let us emphasize that the result of eq. (30) is valid only within the plane-wave approximation and neglecting possible partial coherence in the source [4,5]. If these conditions hold, the asymmetry parameter $-1 \leq \beta \leq 1$ can be determined from the relative momentum dependence of the normalized threeparticle cumulant correlation function $w$, which is denoted also $[32,33]$ as

$$
w=\cos (\phi)
$$

The angle $\phi$ is directly proportional to $\beta$, the asymmetry parameter of the stable distributions, and the coefficents of proportionality can be determined from the two-particle correlation data. Thus we have proven, that for a onedimensional Lévy stable distribution the asymmetry parameter of the source 
can in principle be determined from a combined use of the two- and threeparticle BEC-s. Thus all the essential parameters of the source density can be reconstructed within this class of density distributions.

We would like to note, that the cases of $\beta=+1$ and $\beta=-1$ are particularly interesting, as in these cases the support of $f(x)$ is one-sided, corresponding to $[\delta, \infty)$ and $(-\infty, \delta]$, respectively. Such kinds of Lévy stable distribitions may have applications e.g. in the description of the temporal processes in the collisions of particles at high energies: particle production cannot start before the initial collision - hence the corresponding time interval has to be limited from below.

After its parameters $\alpha, \beta$ and $\gamma$ are determined, a Lévy stable density distribution $f(x)$ can be straightforwardly evaluated using public domain numerical packages [36]. In most of the cases these functions are also known in closed form. The difficulty is that they can be expressed in terms of very general kind of functions, Fox's H-functions, see Schneider's papers for greater details $[37,38]$.

In this sub-section we neglected a possible core-halo correction, because it is straightforward to shown that it cancels from the final result on $w$, eq. (30).

Note, that in the more general case of expanding sources, Bose-Einstein correlations are sensitive only to the so called lengths of homogeneity, which correspond to the local density distribution for particles that have a given momentum $k$. In order to reconstruct the complete picture of the density distribution for all particles, one has to perform a combined analysis of the single particle spectra and the two-particle BEC-s $[16,39,5]$.

For such type of expanding sources, the radius parameters are known to decrease with increasing (transverse) mass of the particles [5,6]. This implies, that the sensitivity of three-particle correlations for the shape parameters of the source distributions is enhanced when the heavier kaons are utilized instead of the pions, as follows if one evaluates eq. (30) using a reduced radius parameter.

\section{Multivariate symmetric Lévy distributions}

Multivariate Lévy stable distributions have properties similar to the univariate case, their study is even presently a research topic in the theory of mathematical statistics, see ref. [40]. In this section, we assume that the source is symmetric, to simplify the presentation. 
Let us denote the coordinate 3 -vector by $\mathbf{x}$, and the time by the variable $t$, while a space-time four-vector is $x=(t, \mathbf{x})$, a momentum space four-vector is $k=(E, \mathbf{k})$.

The simplest case corresponds to a static (non-expanding) source, that emits all the particles instantaneously. This is described by a factorized form of the emission function,

$$
S(x, k)=f(\mathbf{x}) g(\mathbf{k}) \delta\left(t-t_{0}\right) .
$$

If the density distribution $f(\mathbf{x})$ is a multivariate stable distribution that features ellipsoidal contour lines, than the reconstruction of the source density distribution becomes a one-dimensional, solvable problem [25].

The corresponding three-dimensional, symmetric, sub-Gaussian Lévy-stable characteristic function is

$$
\tilde{f}(\mathbf{q})=\exp \left(i \mathbf{q} \mathbf{x}_{\mathbf{0}}-\frac{1}{2}\left|\sum_{i, j=1}^{3} R_{i, j}^{2} q_{i} q_{j}\right|^{\frac{\alpha}{2}}\right)
$$

where $\mathbf{x}_{0}$ stands for the center of particle emission, and $R_{i, j}^{2}$ corresponds to a symmetric matrix $R_{i, j}=R_{j, i}$ with dimensions of radius squared; $\alpha$ is the index of stability similarly to the one-dimensional case, and $\mathbf{q}=\mathbf{k}_{1}-\mathbf{k}_{2}$ stands for the relative momentum.

Again, we have applied a rescaling as compared to the $S(\alpha, 0, \gamma, 0 ; 1)$ class of sub-Gaussian distributions, that are discussed in ref. [25]. The rescaling corresponds to the application of eq. (15) interpreting that equation in a matrix notation. We chose a notation that is adopted to the convention used in correlation studies in high energy physics, so that the Gaussian limiting case, $\alpha=2$, corresponds to the

$$
C(\mathbf{q})=1+\exp \left(-\sum_{i, j} R_{i, j}^{2} q_{i} q_{j}\right)
$$

conventional multivariate Gaussian forms.

Hence, for symmetric, sub-Gaussian multivariate Lévy distributions, the twoparticle BEC has the following simple form

$$
C(\mathbf{q})=1+\exp \left(-\left|\sum_{i, j=1,3} R_{i, j}^{2} q_{i} q_{j}\right|^{\frac{\alpha}{2}}\right)
$$


The components of the squared radius matrix are symmetric, $R_{i j}^{2}=R_{j i}^{2}$. The components of this matrix and that of the relative momentum can be determined in the experimentalists' favourite reference frame. The point is that $\sum_{i, j} q_{i} R_{i j}^{2} q_{j}$ is a (non-relativistic) bilinear form, which is (non-relativistically) invariant for the choice of the coordinate system, hence $\mathbf{q} \mathbf{R}^{2} \mathbf{q}$ can be decomposed in any frame.

The ellipsoids are characterized by the inverse of the $R^{2}$ matrix, which is denoted symbolically by $R^{-2}$, and frequently called the covariance matrix:

$$
\sum_{j} R_{i j}^{2} R_{j k}^{-2}=\delta_{i k}
$$

The large distance behaviour of the density distribution can be analytically determined as follows:

$$
f(\mathbf{x}) \approx\left(\sum_{i j} R_{i j}^{-2} x_{i} x_{j}\right)^{-\frac{1+\alpha}{2}}
$$

so the source density decreases as a power-law for sufficently large values of the arguments, with $0<\alpha<2$. (The case of $\alpha=2$ corresponds to Gaussian source distributions).

The elliptically contoured density distributions can be calculated by integrating a one-dimensional distribution [25]. The ellipsoidal contour lines can be transformed to spherical contours by realizing that the two-particle BECF is characterized by $R^{2}$, a symmetric matrix $\left(R^{2}\right)_{i j}=\left(R^{2}\right)_{j i}$, which always can be written as $R^{2}=R \cdot R^{T}$. The matrix $R$ can be considered as a sort of 'square root' of the squared radius matrix. Physically, the matrix $R$ gives the orientation of the prinicipal axis of the ellipsoids of the density profile in coordinate space, and characterizes also the stretchings along the principal axis that are needed to transform a unit sphere to an ellipsoid corresponding to the contour lines. Hence the bilinear form in the exponent of the BECF can also be written as $\mathbf{q} \mathbf{R}^{2} \mathbf{q}=|\mathbf{R q}|^{2}$.

The density distribution is expressed, in $d>1$ dimensions, for $\alpha<2$, by eqs. (1) and (6) of ref. [34],

$$
\begin{aligned}
f_{\alpha}(s(\mathbf{x})) & =\frac{1}{\left(2 \pi \operatorname{det} R^{2}\right)^{d / 2}} \int_{0}^{\infty} \mathrm{d} t t^{d-1}(t s(\mathbf{x}))^{1-d / 2} J_{d / 2-1}(t s(\mathbf{x})) e^{-t^{\alpha}} \\
s(\mathbf{x}) & =\left|R^{-1} \mathbf{x}\right|
\end{aligned}
$$

where $J_{i}(z)$ is the $i^{\text {th }}$ order Bessel function of the first kind, the matrix $R^{-1}$ is the inverse of the radius matrix $R$, and the function $s(\mathbf{x})$ corresponds to 
a scaling variable that is constant over the ellipsoidal surfaces characterizing the constant source densities. It is relatively easy to see the physical meaning of this equation in $d=3$ dimensions, in the system of ellipsoidal expansions, where the radius matrix corresponds to $R=\operatorname{diag}(X, Y, Z)$, its inverse is $R^{-1}=$ $\operatorname{diag}(1 / X, 1 / Y, 1 / Z)$ and the scaling variable of the ellipsoidally symmetric source distribution is given by

$$
s(\mathbf{x})=\left|R^{-1} \mathbf{x}\right|=\sqrt{\frac{r_{x}^{2}}{X^{2}}+\frac{r_{y}^{2}}{Y^{2}}+\frac{r_{z}^{2}}{Z^{2}}} .
$$

Note that such scaling variables arise naturally in ellipsoidally symmetric solutions of fireball hydrodynamics, that may correspond to non-central collisions of heavy ions in the non-relativistic energy domain [41-43].

In addition to the above analytic result, eq. (38), the case of multivariate symmetric Levy laws is discussed in great details in refs. [34] and [35]. [We don't recapitulate here the well-known results for multivariate Fourier-transforms of Gaussian densities, corresponding to the case of $\alpha=2$.] From the point of view of the applications, it is important that public domain software packages are available [36], that can be utilized to plot the density distribution of stable laws as a function of the scaling variable $s$, if the parameter $\alpha$ is determined from a multi-dimensional fit to the two-particle BEC-s.

Multi-dimensional BEC-s are frequently studied in high energy particle and heavy ion physics. For the sake of completeness, and the applicability of our results to these cases, we give the two-particle BECF in a general form, using the Wigner-function formalism of refs. [44,13,5].

\subsection{Wigner-function formalism}

From now on, $x$ and $k$ stand for four-vectors. Using this notation, most of the results for the univariate case can be generalized to the relativistic case.

In the Wigner-function formalism, the particle emission is characterized by the source or emission function $S(x, k)$. In the non-relativistic limit, this function corresponds to the time-derivative of the Wigner function, which itself is the quantum mechanical analogy to the classical phase-space distribution and this $S(x, k)$ can also be considered as a relativistic, covariant form of the Wigner function.

The invariant momentum distribution and the two-particle BEC can be expressed in the Wigner-function formalism as follows: 


$$
\begin{aligned}
N_{1}\left(k_{1}\right) & =\int \mathrm{d}^{4} x S\left(x, k_{1}\right), \\
C_{2}\left(k_{1}, k_{2}\right) & =1+\frac{|\tilde{S}(q, K)|^{2}}{\tilde{S}\left(0, k_{1}\right) \tilde{S}\left(0, k_{2}\right)} \approx 1+\frac{|\tilde{S}(q, K)|^{2}}{|\tilde{S}(0, K)|^{2}}, \\
\tilde{S}(q, K) & =\int \mathrm{d}^{4} x \exp (i q x) S(x, K), \\
K & =\frac{1}{2}\left(k_{1}+k_{2}\right), \quad q=k_{1}-k_{2} .
\end{aligned}
$$

By introducing the notation

$$
f_{K}(x)=\frac{S(x, K)}{\int \mathrm{d}^{4} x S(x, K)},
$$

the results of the earlier section are recovered, but with all the parameters of the effective source depending on the mean momentum $K$.

\subsubsection{Core-halo correction}

If some of the particles are emitted from the decays of very long lived resonances, such that their decay width is much smaller than the typical 5-10 MeV width of the first bin of the experimentally determined two-particle BECF, then the decay products of these particles contribute to a halo that cannot be resolved with the help of two-particle BEC measurements [1]. Within the core-halo picture, their effects can be taken into account by assuming that the single-particle emission function can be written as a sum of a resolvable core contribution and an unresolvable halo contribution,

$$
S(x, k)=S_{c}(x, k)+S_{h}(x, k)
$$

and as a result, an intercept parameter $\lambda$ is introduced to the two-particle $\mathrm{BEC}$,

$$
C_{2}\left(k_{1}, k_{2}\right) \approx 1+\lambda \frac{\left|\tilde{S}_{c}(q, K)\right|^{2}}{\left|\tilde{S}_{c}(0, K)\right|^{2}},
$$

where the effective intercept parameter $0 \leq \lambda \equiv \lambda(\mathbf{k}) \leq 1$ is interpreted as a (mean-momentum dependent) measure of the fraction of bosons emitted directly from the core:

$$
\lambda=\left[\frac{N_{c}(K)}{N_{c}(K)+N_{h}(K)}\right]^{2},
$$


and the emission function of the core determines the relative-momentum dependence of the BECF.

This formalism is relevant in case of correlation studies for expanding systems. Assuming that the local regions of homogeneity can be characterized by a symmetric multivariate Lévy stable distribution, the general form for the twoparticle BECF is as follows:

$$
C_{2}\left(k_{1}, k_{2}\right)=1+\lambda \exp \left[-\left(\sum_{i, j=1}^{3} R_{i j}^{2} q_{i} q_{j}\right)^{\alpha / 2}\right],
$$

where all the parameters may depend on the mean momentum of the pair, $\left(\lambda, R_{i j}^{2}, \alpha\right)=\left(\lambda(\mathbf{k}), R_{i j}^{2}(\mathbf{K}), \alpha(\mathbf{K})\right)$.

It is at this point where the large $q$ behaviour of the Fourier transformed core density distribution becomes essential: within the core-halo picture, the exact intercept parameter of the two-particle BECF is $\lambda_{\mathrm{xct}}=C_{2}(k, k)-1=1$, however, it is assumed that the halo of long lived resonances creates an unresolvable peak in the $q<q_{\text {min }}$ region, where the two-particle relative momentum resolution is typically $q_{\text {min }} \sim 5 \mathrm{MeV} / \mathrm{c}$. This peak makes the analytic expansions around $q=0$ impossible, and the large $q$ behaviour of the core correlator becomes important. Fortunately, this is uniquely determined with the help of the index of stability $\alpha$ for the chosen class of stable distributions.

The resulting BEC-s all have a generalized exponential form, but, as compared to the usual Gaussian case, there exist an additional scale transformation $q_{i} \rightarrow q_{i}^{\alpha / 2}$ which sometimes is also referred to as stretching. Hence, the resulting correlation functions are multivariate stretched exponential parameterizations. For $\alpha=2$, the earlier, Gaussian forms are recovered, while for $\alpha<2$ the correlation function becomes more peaked than a Gaussian, and it develops longer tails as well. As such a peaked, but almost Gaussian behaviour is frequently seen in the experimentally determined Bose-Einstein correlation data in high energy particle and heavy ion induced reactions, we may hope that the above stretched exponential or sub-Gaussian parameterization provide a useful tool for the characterization of such datasets even in the case of the multi-dimensional analysis.

It follows from the above discussion, that perhaps the presentation of the twoparticle Bose-Einstein correlation data in the usual, linear-linear plot of $C(q)$ versus $q$ is not particularly useful. In order to exhibit the possible power-law structure of the exponent of the correlator, a log-log plot of the two-particle cumulant correlation function, $\ln \{\ln [C(q)-1]-\ln \lambda\}$ versus $\ln q$ would be the most appropriate. On such plots, the two-particle correlation functions would appear as straight lines and the slope parameter of the lines would be given by the index of stability $-\alpha$. 
Below let us give two examples, where we highlight the essential model assumptions and show the result. For more detailed discussion on these topics, see refs. $[5,6]$.

\subsection{Lévy stable distributions for non-relativistic, expanding sources}

Let us consider a popular, but not boost-invariant parameterization of the two-particle BEC-s, the Bertsch-Pratt $[45,46]$ or side, out, long decomposition, where $\mathbf{q}=\left(q_{\mathrm{s}}, q_{\mathrm{o}}, q_{\mathrm{l}}\right)$, where $q_{l}=k_{1, z}-k_{2, z}$ is the longitudinal, $q_{\mathrm{o}}=\left(q_{x} K_{x}+\right.$ $\left.q_{y} K_{y}\right) / \sqrt{K_{x}^{2}+K_{y}^{2}}$ is the out(ward) and $q_{s}=\left(q_{x} K_{y}-q_{y} K_{x}\right) / \sqrt{K_{x}^{2}+K_{y}^{2}}$ is the side(ward) relative momentum komponent. This decomposition is frequently studied in the LCMS frame [47], where $K_{z}=0$, because in this frame, the temporal component of the source couples only to the out direction, see e.g. [5] for details. This can be shown by utilizing the mass-shell constraints for the four-momenta, from which it follows that in the LCMS

$$
E_{1}-E_{2}=\frac{\mathbf{k}_{1}+\mathbf{k}_{2}}{E_{1}+E_{2}}\left(\mathbf{k}_{1}-\mathbf{k}_{2}\right)=\beta_{o} q_{o}
$$

If correlations between coordinate and time are negligible, a model assumption may be that the emission function at every value of the particle momenta can be written as a factorized coordinate-space distribution and a temporal distribution,

$$
S(x, \mathbf{k})=S(\mathbf{x}, \mathbf{k}) h(t)
$$

and we may assume that at every value of $\mathbf{k}$ the coordinate space distribution corresponds to a three-dimensional, sub-Gaussian Lévy distribution with index of stability $\alpha_{x}$, while the temporal distribution would correspond to a univariate Lévy distribution with index of stability $\alpha_{t}$, and in general these two indexes may be different from each other.

Such kind of factorization assumptions can be approximately made not only for non-relativistic expanding sources but sometimes also for relativistic expanding sources when the emission function is evaluated in the LCMS frame [47], and the motion is non-relativistic with respect to this frame.

The resulting two-particle BECF for cylindrically symmetric sources can be written as

$$
C_{2}\left(\mathbf{k}_{1}, \mathbf{k}_{2}\right)=1+\lambda \exp \left[-\left(\sum_{i, j=s, o, l} R_{i j}^{2} q_{i} q_{j}\right)^{\frac{\alpha_{x}}{2}}-\left(\Delta t^{2} \beta_{o}^{2} q_{o}^{2}\right)^{\frac{\alpha_{t}}{2}}\right]
$$


An important feature of eq. (52) is that the spatial and the temporal scales may enter the relative momentum dependence in the out direction with different powers, $\alpha_{x}$ and $\alpha_{t}$, hence, a detailed shape analysis of the BECF in terms of $\mathrm{BP}$ variables can be utilized to extract these different indexes as well as the information on both the spatial and the temporal scales. However, as is well known, the $\alpha_{t}=\alpha_{x}$ case becomes degenerate, and only the combination $R_{o}^{2}+\beta_{o}^{2} \Delta t^{2}$ can be determined from the data analysis in this exactly Gaussian case.

A more realistic case is to include possible correlations between production time and position, which goes beyond the above factorized ansatz.

\subsection{Lévy stable distributions for the invariant Buda-Lund variables}

For collisions with very high energy, the particle emission process becames a highly relativistic phenomena. In this case, the invariance of the emission function can be reflected if the longitudinally boost-invariant proper-time variable $\tau=\sqrt{t^{2}-r_{z}^{2}}$ is utilized, and the space-time rapidity $\eta=0.5 \log \left[\left(t+r_{z}\right) /\left(t-r_{z}\right)\right]$ is also introduced as a hyperbolic, boost-additive coordinate.

In a factorized form the Buda-Lund (BL) parameterization assumes the following structure for the emission function [16]:

$$
S(x, k)=H_{*}(\tau) G_{*}(\eta) I_{*}\left(r_{x}, r_{y}\right),
$$

where the subscript * denotes an implicit momentum dependence. The effective proper-time and space-time rapidity distributions $H_{*}(\tau)$ and $G_{*}(\eta)$ are assumed to have a uni-variate Lévy distributions with indexes of stability $\alpha_{=}$ and $\alpha_{\|}$, while $I_{*}\left(r_{x}, r_{y}\right)$ may have a bivariate Lévy distribution with index $\alpha_{\perp}$. Here we refer to the symbolic notation of the invariant temporal and the parallel relative momentum differences, $Q_{=}$and $Q_{\|}$, being conjugated variables to the space-time variables $(\tau, \eta)$.

The corresponding factorized Lévy-stable BL parameterization of the twoparticle BECF reads as

$$
\begin{aligned}
C\left(k_{1}, k_{2}\right) & =1+\lambda \exp \left(-\left|R_{=} Q_{=}\right|^{\alpha_{=}}-\left|R_{\|} Q_{\|}\right|^{\alpha_{\|}}-\left|R_{\perp} Q_{\perp}\right|^{\alpha_{\perp}}\right), \\
Q_{=} & =m_{t, 1} \cosh \left(y_{1}-\bar{\eta}\right)-m_{t, 2} \cosh \left(y_{2}-\bar{\eta}\right) \\
Q_{\|} & =m_{t, 1} \sinh \left(y_{1}-\bar{\eta}\right)-m_{t, 2} \sinh \left(y_{2}-\bar{\eta}\right) \\
Q_{\perp} & =\sqrt{Q_{x}^{2}+Q_{y}^{2}} .
\end{aligned}
$$


In these equations, $m_{t, i}=\sqrt{m^{2}+\mathbf{k}_{i}^{2}}$ is the transverse mass, $y_{i}=0.5 \ln \left[\left(E_{i}+\right.\right.$ $\left.\left.k_{z, i}\right) /\left(E_{i}-k_{z, i}\right)\right]$ is the rapidity of particle $i$ and $\bar{\eta}$ stands for the space-time rapidity of the point of maximum emissivity for particles with a given fixed four-momentum $k_{i}$. Note that the above equations for the definition of the temporal and parallel invariant momentum differences are equivalent to the ones given in ref. [5], but the invariant decomposition is perhaps more apparent in the above form.

The three different indexes of stability, $\alpha_{=}, \alpha_{\|}$and $\alpha_{\perp}$ appear as new fit parameters, due to the assumed factorization property of the boost-invariant temporal, longitudinal and transverse distributions from each other. All are Lévy indexes for the corresponding spatial distributions, hence satisfy the usual unequality $0<\alpha_{i} \leq 2$ for all ${ }_{i}=(=,\|\|$,$) .$

All the five scale parameters, $\lambda, R_{=}, R_{\|}, R_{\perp}$ and $\bar{\eta}$ may depend on the value of the mean momentum $K$, due to the expanding nature of the particle emitting source.

\section{Applications to NA22 and UA1 data}

The case of the uni-variate stable distributions can be checked, relatively easily, against the precision data on the two-particle short-range correlation functions as measured in the CERN experiments NA22 [48] and UA1 [49].

We emphasize, that we are not attempting here to reconstruct the detailed space-time structure of the particle emission process, or, interpret the result in terms of a physical process that may result a Lévy stable law. We merely consider this application as a first test of the feasibility of our method as a quantitative tool for the analysis of two-particle BEC in high energy physics.

The parameters for the best Lévy stable fits are summarized in Table 1.

The two-particle BEC for Lévy stable distributions is a stretched exponential. Hence the title of Figure 1 where the best Lévy stable fits are shown for both the NA22 and the UA1 data sets.

The plots of the best Lévy fits are clearly better than even the best exponential fits to the same curve (not mentioning the "best Gaussian" approximations, who miss completely the strongly rising peak near to the $Q=0$ point).

The quality of the fits is approximately similar to those obtained with the help of Laguerre expansions. However, an advantage of the Lévy stable laws and fits is that they relate the exponent $\alpha$ to the power-law tail behaviour of 
density fluctuations in the large coordinate-space distributions of elementary particles. We discuss a possible interpretation of our result in terms of QCD cascades in a separate paper [24].

The following statements can be made without any reference to a dynamical model:

Both data sets can be described by the Lévy stable fits in a statistically acceptable manner. In case of the NA22 data, the confidence level of the fit is about $80 \%$ even when one of the fit parameters is fixed, as determined from a Laguerre expansion method in ref. [10]. Without fixing this parameter, the minimum was found to be degenerate and the confidence level even higher. In case of the UA1 data, the confidence level of the fit is only $6 \%$, a rather low value.

In case of the NA22 data set, the fitted value of the $\lambda$ parameter is within errors close to the maximum value of 1 which is a theoretical upper limit within the core-halo picture. Such an upper limit is violated by the fit to the UA1 data set. This may indicate that in the UA1 data there some non-BoseEinstein type of short range correlations present. However, fixing $\lambda=1$ a Lévy stable fit to the UA1 data becomes still possible, but only with a small a confidence level of about $1 \%$. Fixing the normalization to that of the Laguerre expansion for the same function, ref. [10], a Lévy stable fit becomes possible with only a very small, $0.1 \%$ confidence level. In all of the above cases, $\alpha$ remains significantly below 1 .

Fixing $\alpha=1$ (exponential fit), the confidence level of the fit to the NA22 data decreases from $80 \%$ to $24 \%$, in case of the UA1 data, from $6 \%$ to $10^{-8}$. Fixing $\alpha=2$ (Gaussian fit), the confidence level of the best fit to the NA22 data decreases to $10^{-8}$, while it becomes essentially 0 in case of the UA1 data set.

The conclusion is that Lévy stable (or stretched exponential) fits describe the NA22 and the UA1 data on short-range correlations of particles in high energy physics. In case of the NA22 data, the confidence level of the fit is very high and the fitted parameters can be interpreted within the core-halo picture, with a fully resolved halo. In case of the UA1 data, the best fits are obtained when the parameters were utilized outside of their usual domain. This result may indicate the presence of short-range correlations of not Bose-Einstein type in the UA1 data set. 


\section{Discussion and conclusion}

Lévy stable laws appear in many branches of physics, mathematics, biology, economy, computer science and other areas, where the scale of fluctuations may be characterized by long tails and an asymptotic power-law like behaviour. As the Lévy stable distributions satisfy a generalized central limit theorem, they may appear naturally also in case of high energy elementary particle physics, where self-similar branching processes are known to exist on the large momentum transfer scale, a self-similar string fragmentation process is expected to describe the distributions of directly produced hadrons, and where the distribution of abundances of various resonances may also follow an approximate power-law like behaviour as a function of the life-time of the resonance. Similar processes are expected to exist also in high energy heavy ion collisions, where additional fluctuations from a hadronic or quark matter may also modify the spectrum of these fluctuations.

We have argued, that additive stochastic processes may determine the spacetime density distribution of elementary production in highly energetic reactions of particles or heavy ions. In this case, if a generalized Central Limit Theorem is applicable, the probability distribution of particle emission corresponds to a(n univariate or multivariate) Lévy stable distribution.

Although the characterization of the Lévy stable distributions is not straightforward, by now these distributions are numerically as well as analytically accessible. Their most important property is that the Fourier transformed emission function has in general a non-analytic structure in the vanishing relative momentum region.

We have shown, that due to this reason, the two-particle Bose-Einstein correlation functions are characterized by a sub-Gaussian two-particle Bose-Einstein correlation function, with an index of stability $0<\alpha \leq 2$. The analytic behaviour and the Gaussian limiting case corresponds to the $\alpha=2$ special case only, a single point out of the uncountably infinite possibilities in the parameter space. Hence there is no reason to believe that the two-particle Bose-Einstein correlation functions have to behave as (multivariate) Gaussian forms.

We have demonstrated, in the simplest one-dimensional case, that the NA22 and the UA1 two-particle Bose-Einstein correlation functions are well described with a Lévy-stable law and the index of stability was found to be significantly smaller than 2, i.e. the deviation from a Gaussian behaviour in this case can be quantified and characterized with our method.

We have also determined the form of the two-particle Bose-Einstein correlation function in case the particle emission is characterized by a multivari- 
ate Lévy-stable law. In particular, we have generalized the non-relativistic Bertsch-Pratt and the relativistically invariant Buda-Lund parameterizations for multivariate Lévy-stable laws.

\section{Acknowledgments}

Cs. T. is indebted to W. Kittel for inspiration, support and careful attention. Without him this manuscript could not have been formulated. He is also greatful to R. Hakobyan and the L3 collaboration for an inspiration to complete this paper, and to J. P. Nolan for enlightening communications. This research has been supported by an NWO - OTKA grant N25487, by the Hungarian NSF grants OTKA (T024094, T026435), T034269, T038406 and T043514, by a NATO Senior Fellowship (Cs. T.), the NATO PST.CLG.980086 grant and by an MTA-OTKA-NSF grant. 


\section{References}

[1] T. Csörgö, B. Lörstad and J. Zimányi, Z. Phys. C71 (1996) 491 hepph/9411307.

[2] S.E. Vance, T. Csörgő and D. Kharzeev, Phys. Rev. Lett. 81 (1998) 2205, nuclth/9802074.

[3] A. Ster and T. Csörgö, PRHEP-hep2001 (2001) 241 [arXiv:hep-ph/0112065], see also

M. Csanád, T. Csörgö, B. Lörstad and A. Ster, arXiv:nucl-th/0403074, J. Phys. $\mathrm{G}$ in press.

[4] T. Csörgő, B. Lörstad, J. Schmid-Sorensen and A. Ster, Eur. Phys. J. C9 (1999) 275 hep-ph/9812422.

[5] T. Csörgő, Heavy Ion Phys. 15 (2002) 1-80, [arXiv:hep-ph/0001233]

[6] W. Kittel, Acta Phys. Polon. B 32 (2001) 3927, [arXiv:hep-ph/0110088]

[7] R. Hanbury Brown and R. Q. Twiss, Phil. Mag. 45, 663 (1954).

[8] R. Hanbury Brown and R. Q. Twiss, Nature 178, 1046 (1956).

[9] G. Goldhaber, S. Goldhaber, W. Y. Lee and A. Pais, Phys. Rev. 120, 300 (1960).

[10] T. Csörgő and S. Hegyi, Phys. Lett. B 489, 15 (2000).

[11] D. Miskowiec and S. Voloshin, Heavy Ion Phys. 9, 283 (1999) [arXiv:nuclex/9704006].

[12] U. W. Heinz and B. V. Jacak, Ann. Rev. Nucl. Part. Sci. 49, 529 (1999) [arXiv:nucl-th/9902020].

[13] U. A. Wiedemann and U. W. Heinz, Phys. Rept. 319, 145 (1999) [arXiv:nuclth/9901094].

[14] R. M. Weiner, Phys. Rept. 327, 249 (2000) [arXiv:hep-ph/9904389].

[15] T. Csörgő and J. Zimányi, Nucl. Phys. A 517, 588 (1990).

[16] T. Csörgő and B. Lörstad, Phys. Rev. C 54, 1390 (1996) [arXiv:hep$\mathrm{ph} / 9509213]$.

[17] S. Nickerson, T. Csörgő and D. Kiang, Phys. Rev. C57 (1998) 3251 [arXiv:nuclth/9712059].

[18] D. Hardtke and S. A. Voloshin, Phys. Rev. C 61, 024905 (2000) [arXiv:nuclth/9906033].

[19]

[20] F. B. Yano and S. E. Koonin, Phys. Lett. B 78 (1978) 556 
[21] V. V. Uchaikin and V. M. Zolotarev, "Chance and Stability, Stable Distributions and Their Applications", VSP Science,1999, ISBN: 90-6764-301-7, 596 pp.

[22] V. M. Zolotarev, "One-dimensional Stable Distributions", Am. Math. Soc. Transl. of Math. Monographs, vol. 65, Providence, R.I. (Transl. of the original 1983 Russian)

[23] J. P. Nolan, Stable Distributions: Models for Heavy Tailed Data http://academic2. american.edu/ jpnolan/stable/CHAP1.PDF

[24] T. Csörgö, S. Hegyi and W. Kittel, manuscript in preparation.

[25] J. P. Nolan, Fitting Data and Assessing Goodness-of-fit with Stable Distributions, Contribution ot the Heavy Tails Conference, http://academic2.american.edu/ jpnolan/stable/DataAnalysis.ps

[26] G. Gustafson and A. Nilsson, Nucl. Phys. B 355 (1991) 106.

[27] Y. L. Dokshitzer and I. M. Dremin, Nucl. Phys. B 402 (1993) 139.

[28] E. A. De Wolf, I. M. Dremin and W. Kittel, Phys. Rept. 270, 1 (1996) [arXiv:hep-ph/9508325].

[29] B. Andersson, G. Gustafson, G. Ingelman, T. Sjöstrand, Phys. Rept. 97 (1983) 31

[30] A. Bialas, Acta Phys. Polon. B 23, 561 (1992).

[31] O. V. Utyuzh, G. Wilk and Z. Wlodarczyk, Phys. Rev. D 61 (2000) 034007

[32] U. W. Heinz and Q. H. Zhang, Phys. Rev. C 56, 426 (1997) [arXiv:nuclth/9701023].

[33] H. Heiselberg and A. P. Vischer, arXiv:nucl-th/9707036.

[34] V. M. Zolotarev, in Contributions to probability, Academic Press, New York, 1981, pp. 283-305

[35] H. Abdul-Hamid and J. P. Nolan, J. of Multivariate Analysis 67 80-89 (1998) ; J. P. Nolan and B. Rajput, Commun. Statistics-Simula. 24 551-556 (1995).

[36] J. P. Nolan, a program to calculate multivariate stable densities; http://academic2.american.edu/ ${ }^{j}$ pnolan/stable/mvstable.exe

[37] W.R. Schneider: Stable distributions: Fox function representation and generalization, in Stochastic Processes in Classical and Quantum Systems (Lecture Notes in Physics, Springer Verlag, Berlin, 1986, eds. S. Albeverio, G. Casati and D. Merlini), Vol. 262, pp. 497-511.

[38] W.R. Schneider: Generalized one-sided stable distributions Proceedings of the second BiBoS Symposium, in: Stochastic Processes - Mathematics and Physics II. (Lecture Notes in Mathematics, Springer Verlag, Berlin, 1986, eds. S. Albeverio, Ph. Blanchard and L. Streit) Vol. 1250 pp. 269-287. 
[39] T. Csörgő and B. Lörstad, Nucl. Phys. A 590, 465C (1995) [arXiv:hep$\mathrm{ph} / 9503494]$.

[40] J. P. Nolan, Multivariate stable distributions: approximation, estimation, simulation and identification, http://academic2.american.edu/ jpnolan/stable/overview.ps

[41] S. V. Akkelin, T. Csörgő, B. Lukács, Y. M. Sinyukov and M. Weiner, Phys. Lett. B 505 (2001) 64 [arXiv:hep-ph/0012127].

[42] T. Csörgő, S. V. Akkelin, Y. Hama, B. Lukács and Y. M. Sinyukov, arXiv:hep$\mathrm{ph} / 0108067$.

[43] T. Csörgö, arXiv:hep-ph/0111139.

[44] S. Pratt, T. Csörgő and J. Zimányi, Phys. Rev. C 42, 2646 (1990).

[45] G. F. Bertsch, Nucl. Phys. A 498, 173C (1989).

[46] S. Pratt, Phys. Rev. Lett. 53, 1219 (1984).

[47] T. Csörgő and S. Pratt, KFKI - 1991 - 28/A, p. 75

[48] N. M. Agababian et al. [EHS/NA22 Collaboration], Z. Phys. C 59, 405 (1993).

[49] N. Neumeister et al. [UA1-Minimum Bias-Collaboration], Z. Phys. C 60, 633 (1993). 
Table 1

Best fits to UA1 and NA22 two-particle correlations using a Lévy stable law

\begin{tabular}{|c|c|c|c|c|c|}
\hline \multirow[b]{2}{*}{ Parameter } & \multicolumn{2}{|l|}{ UA1 } & \multicolumn{3}{|c|}{ NA22 } \\
\hline & Value & Error & & Value & Error \\
\hline $\mathcal{N}$ & 1.34 & \pm 0.03 & 0.95 & (fixed) & \\
\hline$\lambda$ & 1.85 & \pm 0.07 & & 1.15 & \pm 0.17 \\
\hline$R[\mathrm{fm}]$ & 6.36 & \pm 0.33 & & 1.33 & \pm 0.30 \\
\hline$\alpha$ & 0.49 & \pm 0.01 & & 0.67 & \pm 0.07 \\
\hline$\chi^{2} / N D F$ & \multicolumn{2}{|c|}{$56.5 / 42=1.35$} & \multicolumn{3}{|c|}{$27.67 / 35=0.769$} \\
\hline CL & \multicolumn{2}{|c|}{$6.6 \%$} & \multicolumn{3}{|c|}{$80.6 \%$} \\
\hline
\end{tabular}


Stretched exponential fit
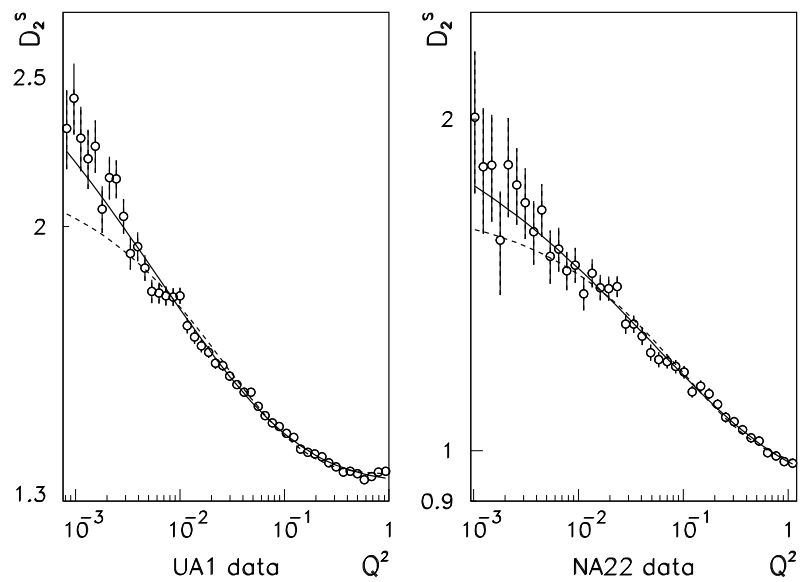

Fig. 1. The figures show $D_{2}^{s}$ which is proportional to the two-particle short range correlation function, as measured by the UA1 and the NA22 collaborations at CERN. The lines stand for the stretched exponential (or Levy) fits, which are able to reproduce the data with an acceptable $\chi^{2} / N D F$, while the dashed lines stand for the result of the best exponential fits. The fit results are summarized in Table 1. 